\title{
XX VIII. \\ Ueber die Reduction des Arseniks und des Antimons aus den Verbindungen dieser Metalle mittelst des Cyankaliums.
}

Von

II. Rose.

(Ber. d. Berl. Akad.)

Das Cyankalium kann bekanntlich durchs Schmelzen mit den Verbindungen mehrerer Metalle letztere in vielen Fällen reduciren. Man kann diese reducirende Eigenschaft des Cyankaliums auf mannigfaltige Weise in der analytischen Chemie benutzen, muss dann aber die Fălle genau kennen, in welchen diese Reduction theilweise und gänzlich verhindert werden kann. Diese hat der Verfasser möglichst genau, zuerst nur boi den Verbindungen des Arseniks und des Antimons, anzugeben versucht.

\section{Arsenikverbindungen.}

Durchs Schmelzen mit Cyankalium wird das Arsenik aus seinen Verbindungen nach der Reduction verflüchtigt. Wegen dieser leichten Verflüchtigung des Metalls kann die Quantităt desselben nicht gut mit Genauigkeit bestimmt werden; aber bei qualitativen Untersuchungen bedient man sich schon seit längerer Zeit des Cyankaliums, um die Gegenwart dieses Mẹtálls mit Sicherheit auch in solchen Verbindungen zu finden, in denen es durch andere Mittel schwieriger zu reduciren ist.

Beide Arten des Schwefelarseniks, $\mathrm{AsS}_{3}$ und $\mathrm{AsS}_{5}$, geben in der kleinsten Menge in einer Glasrőhre mit Cyankalium geschmolzen einen Spiegel von metallischem Arsenik. Es bildet sich dadurch Rhodankalium, aber die ganze Menge des Arseniks wird durch Cyankalium nicht aus dem Schwefelmetall reducirt, und verflüchtigt. Es erzeugt sich ein Schwefelsalz des Arseniks, in welchem das Schwefelarsenik der Zersetzung durch Cyankalium widersteht. Wird die geschmolzene Masse daher in Wasser gelőst, so wird aus der Losung durch verdünnte Säuren unter Entwicklung von Schwefelwasserstoff gelbes Schwefelarsenik gefäll. 
Mengt man daher Schwefelarsenik mit Schwefel, so wird aus dem Gemenge durchs Schmelzen mit Cyankalium kein Arsenik abgeschieden, und kein metallischer Spiegel erzeugt.

Aber auch die Gegenwart von leicht reducirbaren Metallen kann die Sublimation des Arseniks und die Abscheidung der ganzen Menge desselben oder auch nur eines Theils verhindern. Das Arsenik scheidet sich dann gemeinschaftlich mit dem reducirten Metalle $a b$, und bildet mit demselben ein Arseniet, aus welchem bei einem gewissen Ueberschuss des Arseniks nur ein Theil desselben sich im metallischen Zustande sublimiren kann.

Wird arsenigsnutcs Kupferoxyd (Scheele's Grün) mit Cyankalium geschmolzen, so erhält man nur einen geringen Spiegel von sublimirtem Arsenik. Wird hingegen das Kupfersalz mit mehr Kupferoxyd innig gemengt, und das Gemenge alsdann mit Cyankalium geschmolzen, so erbălt man keine Spur von einem sublimirten Arsenikspiegel.

Mengt man arseniksaures Natron mit einem Ueberschuss von Bleioxyd und schmelzt das Ganze mit Cyankalium, so reducirt sich zwar die ganze Menge des Arseniks und zugleich das Blei, aber es zeigt sich kein sublimirtes Arsenik.

Durchs Schmelzen von arseniksaurem Bleioxyd mit Cyankalium erhält man viel sublimirtes reducirtes Arsenik. Mengt man aber das Salz vorher mil vielem Bleioxyd, so kann durchs Schmelzen mit Cyankalium kein Arsenikspiegel erhalten werden.

Wird fein zertheiltes Schwefelblei mit Schwefelarsenik $\left(\mathrm{AsS}_{3}\right)$ gemengt, mit Cyankalium geschmolzen, so erhält man einen, wiewohl schwachen Spiegel von Arsenik, selbst wenn Schwefelblei in einem bedeutenden Ueberschuss vorhanden ist. Wird aber vorher das Schwefelblei mit dem Schwefelarsenik zusammengescbmolzen, oder nur so stark erhitzt, dass beide zusammensintern, so zeigt sich beim Schmelzen mit Cyankalium kein sublimirtes Arsenik.

Eben so wird die Erzeugung von sublimirtem Arsenik verhindert, wenn arseniksaures Natron gemeinschaflich mit Cyankalium, mit sehr vielem Silberoxyd, mit Gold, mit Eisenoxyd, mit Nickeloxyd und mit Kobaltoxyd zusammengeschmolzen wird. 
Wird hingegen Manganoxyd oder Manganoxyd-Oxydul mit arseniksaurem Natron gemengt, der Schmelzung mit Cyankalium unterworfen, so erhālt man einen starken Spiegel von sublimirtem Arsenik. Es vermag aber das Cyankalium durch Schmelzen das Mangasoxyd nicht zu reduciren.

Aus demselben Grunde zeigt sich auch ein starker Spiegel von sublimirtem Arsenik, wenn arseniksaures Natron mit vielem Zinkoxy a gemengt, mit Cyankalium zusammengeschmolzen wird. Eine Legirung von Zink mit wenig Arsenik indessen giebt mit Cyankalium geschmolzen kein metallisches Arsenik.

Wismuthoxyd auch in sehr bedeutender Nenge mit arseniksaurem Natron und Cyankalium geschmolzen, kann die Verflücbtigung des reducirten Arseniks nicht verhindern, obgleich das Wismuthoxyd gänzlich dabei reducirt 'wird. Aber die Verwandtschaft des Wismuths zum Arsenik ist eine so schwache, dass durch blosse Erhitzung das Arsenik aus einer Legirung von Arsenik und Wismuth ausgetrieben werden kann.

Von keinem Metall aber lässt sich das Arsenik so vollständig durch blosse Erbitzung trennen, wie vom Antimon. Deshalb kann man in allen Antimon-Verbindungen auf keine andere Weise so sicher einen sehr geringen Gehalt von Arsenik auffinden, wie auf die, dass man sie mit Cyankalium schmilat. Die kleinste Nenge von Arsenik wird nach der Reduction verflüchtigt, und es bleibt nichts davon bei dem zu gleicher Zeit reducirten Antimon.

Auch in dem Schwefelantimon lässt sich durch Cyankalium ein sebr kleiner Gehalt von Schwefelarsenik mit Leichtigkeit ent.decken. Der Verf. weist aber ausführlich nach, weshalb auf diese Weise in dem im Handel vorkommenden Antimonium crutum, das fast immer kleine Mengen von Schwefelarsenik enthält, ler Arsenikgehalt nicht aufzufinden ist.

In den Yerbindungen der Arseniksüure mit den Alkalien, Jen alkalischen Erden, der Magnesia und der Thonerde lässt sich durch Cyankalium das Arsenik leicht reduciren und kann als metallischer Spiegel erhalten werden. 


\section{Antimonverbindungen.}

In den antimonsauren Alkalien wird durchs Schmelzen mit Cyankalium das Antimon vollständig reducirt, und nach Auflösung der geschmolzenen Masse in Wasser fast ganz als eine grosse Kugel erhalten; nur eine sehr geringe Menge des Metalls wird pulverförmig abgeschieden. Man kann indessen bei quantitativen Analysen die Reduction des Antimons vermittelst des Cyankaliums zur Bestimmung dieses Metalls nicht anwenden, weil eine kleine Mlenge desselben sich während des Schmelzens verflüchtigt. Es geschieht dies wahrscheinlich durch die Feuclligkeit des Cyankaliums als Antimonwasserstoff.

Auch aus Scliwefelantimon, und zwar aus allen Modificationen desselben, wird durch Schmelzen mit Cyankalium das Antimon reducirt, aber nicht vollständig. Es bildet sich Rhodankalium. Wird aber die geschmolzene Salzmasse in Wasser gelüst, und die filtrirte Lösung durch verdünnte Chlorwasserstoffsünre übersättigt, so scheidet sich unter Entwicklung von Cyanwasserstoff- und von Schwefelwasserstoffgas rothes Scliwefelantion on ab. Es bildet sich beim Schmelzen neben Rhodankalium ein Scliwefelsalz des Antimons, aus welchem durch Cyankalium das Antimon nicht reducirt abgescbieden werden sann.

Aus einem Schwefelsalze des Antimons, z. B. aus der bekannten Verbindung von Antimonsulphid und Schwefelnatrium $\left(3 \mathrm{NaS}+\mathrm{SLS}_{5}\right)$ wird daher durch Cyankalium gar kein Antimon motallisch. abgeschieden. Ebęn so wenig geschieht dies auch, wemn man kohlensaures und antimonsaures Alkali, Schwelel und Cyankalium zusammenschmelzt. 